\title{
EFEITO DA HIPERTERMIA NA PANCREATITE AGUDA GRAVE EXPERIMENTAL
}

\section{EFFECTS OF HYPERTHERMIA ON EXPERIMENTAL SEVERE ACUTE PANCREATITIS}

\author{
Maurício Gustavo Ieri Yamanari ${ }^{1}$; Tiago Alexandre Kunitake ${ }^{1}$; José Lui z Jesus de Almeida ${ }^{1}$; \\ José Jukemura² ; José Eduardo Monteiro da Cunha, TCBC-SP ${ }^{3}$; Marcel Cerqueira César Machado, TCBC-SP ${ }^{4}$
}

\begin{abstract}
RESUMO: Objetivo: O objetivo deste estudo é avaliar os efeitos da hipertermia na pancreatite aguda (PA) grave experimental induzida por ácido taurocólico. Método: A PA grave foi induzida pela injeção retrógrada de ácido taurocólico a 2,5\% ou 5\% no ducto pancreático principal. Após a indução, os animais foram colocados numa gaiola contendo duas lâmpadas de $100 \mathrm{~W}$. A temperatura corporal foi aumentada para $39,5^{\circ} \mathrm{C}$ e mantida neste nível por 45 minutos. Foram estudados taxa de mortalidade em 72 horas, permeabilidade vascular no pâncreas, porcentagem de água no tecido pancreático, amilase sérica, histologia (edema, necrose acinar e infiltrado inflamatório) e níveis séricos de IL-6 e IL-10. Resultados: Não houve alteração em nenhum dos parâmetros avaliados. Conclusão: Não há benefício da hipertermia na PA grave experimental induzida por ácido taurocólico (Rev. Col. Bras. Cir. 2007; 34(1): $35-40)$.
\end{abstract}

Descritores: Pancreatite; Doença aguda; Inflamação; Hipertermia induzida.

\section{INTRODUÇÃO}

A pancreatite aguda (PA) é uma doença com expressão clínica variável. A maioria dos casos $(85-95 \%)$ se apresenta como doença auto-limitada, porém em alguns pacientes ela pode se manifestar sob forma grave, com altos índices de complicações e mortalidade ${ }^{1-3}$.

A gravidade e o prognóstico da doença estão diretamente relacionados à intensidade do dano tecidual ${ }^{4}$. Nos casos de lesão pancreática grave, o paciente pode evoluir com disfunções respiratórias, cardiovasculares, renais e imunológicas. Nesta situação, a mortalidade pode variar de $10 \mathrm{a}$ $100 \%$, sendo maior nos casos em que ocorre necrose e infecção pancreática. Esses pacientes necessitam de cuidados intensivos por longo período e múltiplos debridamentos cirúrgicos ${ }^{3}$.

A fisiopatologia de inflamação pancreática ainda não é totalmente compreendida, entretanto, sabe-se que após a ativação das enzimas digestivas intracelulares, há resposta inflamatória proporcional à gravidade das lesões ${ }^{2,5}$.

Evidências sugerem que citocinas pró-inflamatórias tais como IL- $1 \beta$, TNF- $\alpha$ e IL- 6 agem como mediadores das manifestações locais e sistêmicas da $\mathrm{PA}^{6-9}$. As células pancreáticas inflamatórias ativadas liberam essas proteínas em resposta ao dano tecidual local. Alguns estudos demonstram que a PA melhora após bloqueio da liberação destas citocinas $^{10}$. Semelhantemente, a temperatura é um importante fator que pode mudar a evolução de mecanismos inflamatórios e a expressão de seus mediadores.

A mortalidade foi mais acentuada nos coelhos infectados por Streptococcus pneumoniae do que naqueles do grupo de controle quando a temperatura dos animais foi aumentada de $1,5^{\circ} \mathrm{C}$ por aquecimento externo ${ }^{11}$. Da mesma forma, o aumento da temperatura central de camundongos para $39,5^{\circ} \mathrm{C}$ modifica a expressão de TNF- $\alpha$ sistêmico em reposta ao lipopolissacáride ${ }^{12}$. Por sua vez, Hammami et al. ${ }^{13}$ mostraram que a hipertermia modifica os receptores solúveis de mediadores inflamatórios em vítimas de insolação. Em resumo, as alterações causadas na resposta inflamatória pela temperatura ainda são controversas.

O presente trabalho tem por objetivo estudar o efeito da hipertermia na pancreatite aguda grave induzida por ácido taurocólico em ratos, avaliando-se a intensidade da inflamação pancreática e a taxa de mortalidade em dois níveis diferentes de gravidade (taurocolato a 2,5 e a $5 \%$ ).

\section{MÉTODO}

Todos os experimentos foram realizados de acordo com os protocolos aprovados pelo Comitê de Ética Local. No total, foram utilizados 100 ratos, machos, da raça Wistar, com peso aproximado de $250 \mathrm{~g}$, provenientes do Laboratório de Investigação Médica 37 (LIM-37), distribuidos em dois grupos principais que foram subdivididos em seis grupos menores:

\section{Grupo I $(\mathrm{n}=50)$}

Ratos com pancreatite aguda induzida por ácido taurocólico e submetidos à hipertermia.

Subgrupo IA $(n=20)$ : PA induzida por ácido taurocólico a $5 \%$. Os animais foram observados por um período de 72 horas, no intuito de se estudar mortalidade.

1. Acadêmicos da Faculdade de Medicina da Universidade de São Paulo.

2. Assistende-Doutor do Serviço de Cirurgia das Vias Biliares e Pâncreas.

3. Diretor do Serviço de Cirurgia das Vias Biliares e Pâncreas.

4. Titular da Disciplna de Transplante e Cirurgia do Fígado.

Recebido em 20/07/06

Aceito para publicação em 21/08/06

Conflito de interesses: nenhum

Fonte de financiamento: nenhuma

Trabalho realizado no Departamento de Gastroenterologia e Departamento de Cirurgia - Faculdade de Medicina da Universidade de São Paulo. 
Subgrupo IB ( $n=20)$ : PAinduzida por ácido taurocólico a $2,5 \%$. Os animais foram observados por um período de 72 horas, no intuito de se estudar mortalidade.

Subgrupo IC ( $\mathrm{n}=10)$ : PAinduzida por ácido taurocólico a 2,5\%. Foram avaliados permeabilidade vascular, conteúdo de água do tecido pancreático, morfologia histológica e os níveis séricos de amilase, interleucina - 6 (IL-6) e interleucina -10 (IL-10).

Grupo II $(\mathrm{n}=50)$

Ratos com pancreatite aguda induzida por ácido taurocólico e mantidos em temperatura ambiente.

Subgrupo IIA $(n=20)$ : PA induzida por ácido taurocólico a $5 \%$. Os animais foram observados por um período de 72 horas, no intuito de se estudar mortalidade.

Subgrupo IIB $(n=20)$ : PA induzida por ácido taurocólico a $2,5 \%$. Os animais foram observados por um período de 72 horas, no intuito de se estudar mortalidade.

Subgrupo IIC $(\mathrm{n}=10)$ : PA induzida por ácido taurocólico a 2,5\%. Foram avaliados permeabilidade vascular, conteúdo de água do tecido pancreático, morfologia histológica e os níveis séricos de amilase, interleucina - 6 (IL6) e interleucina-10 (IL-10).

A indução de PA grave foi realizada conforme previamente descrito por Lanckish et al. ${ }^{14}$. Os ratos foram submetidos a anestesia geral com cloridrato de cetamina (Ketalar, Parke-Davis, São Paulo, Brasil) na dose de $100 \mathrm{mg} / \mathrm{Kg}$, por via intraperitoneal, e permaneceram sedados até o sacrifício, exceto os animais que foram observados para se determinar a taxa de sobrevida em 72 horas, os quais não permaneceram sedados após o término do efeito anestésico._Imediatamente após a injeção de taurocolato no ducto pancreático, foi administrado dose do corante Azul de Evans através da veia dorsal do pênis nos animais dos grupo IC e IIC.

$\mathrm{O}$ aquecimento foi obtido com os ratos em decúbito lateral, sob sedação, com a utilização de gaiola com duas lâmpadas $100 \mathrm{~W}$ parcialmente termo-isoladas, durante o período de uma hora. Os animais foram monitorados por termômetro retal e mantidos a temperatura de $39,5^{\circ} \mathrm{C}$. O tempo médio para se atingir tal temperatura foi de 15 minutos.

$\mathrm{O}$ aquecimento foi iniciado logo após a indução de PA com taurocolato. Todos os animais dos grupos que sofreram aquecimento receberam $5 \mathrm{ml}$ de solução salina por via subcutânea imediatamente após o término da indução da PA. Tal medida teve como objetivo assegurar adequada hidratação do animal durante o período de hipertermia.

Logo após o término do período de hipertermia, os ratos dos subgrupos IC e IIC foram, sob anestesia geral, submetidos a laparotomia mediana e punção cardíaca para coleta de sangue.

O pâncreas foi exposto, após tração do cólon e pinçamento da veia porta, e removido na sua totalidade em duas porções, à direita da veia porta, ou proximal ao duodeno, e à esquerda da veia porta, ou distal ao duodeno, e dissecado cuidadosamente, retirando-se todo o tecido adiposo e linfonodos adjacentes. Os animais destes grupos foram sacrificados por exsanguinação.
A amilase sérica nos animais do subgrupo IC e IIC foi dosada pelo método colorimétrico de Bernfeld et $a l^{15} \mathrm{e}$ modificado por Jamieson et al. ${ }^{16}$. Neste método, o amido solúvel $(10 \mathrm{mg} / \mathrm{ml})$ em tampão fosfato $0,02 \mathrm{M} \mathrm{pH} \mathrm{6,9} \mathrm{é} \mathrm{utilizado}$ como substrato, o ácido 3,5 dinitrosalicílico como reagente de cor e a maltose como padrão $(1 \mathrm{mg} / \mathrm{ml})$. Os resultados são expressos em $\mathrm{mg} / \mathrm{ml} / \mathrm{min}$.

Nos ratos dos subgrupos IC e IIC foram coletadas amostras de sangue imediatamente após o período de hipertermia ou normotermia para dosagem dos seguintes mediadores: interleucina-6 (IL-6) e interleucina-10 (IL-10).

As amostras (sangue) foram colhidas e imediatamente centrifugadas a $3000 \mathrm{~g}$ por 10 minutos a $0^{\circ} \mathrm{C}$ e os sobrenadantes estocados a $-20^{\circ} \mathrm{C}$. Os resultados foram expressos em $\mathrm{pg} / \mathrm{ml}$.

A determinação quantitativa dos mediadores foi realizada por Ensaio de Imunoabsorção Ligado à Enzima (ELISA) através da utilização dos kits da Biosource International Cytoscreen $^{\mathrm{TM}}$ conforme manual do fornecedor.

$\mathrm{O}$ pâncreas foi imediatamente pesado em balança analítica após a sua extração (peso fresco), em seguida colocado em estufa a $56^{\circ} \mathrm{C}$ por 48 horas para desidratação (peso seco).

O conteúdo de água do pâncreas foi calculado segundo a fórmula:

\section{[ (peso fresco - peso seco) / peso fresco ] x 100}

e expresso em porcentagem do peso total.

Permeabilidade Vascular do Pancreas Avaliada Pelo Extravasamento do Azul de Evans (Subgrupos IC e IIC).

O pâncreas foi colocado em tubo de ensaio com $3 \mathrm{ml}$ de formamida, na dose de $4 \mu \mathrm{g} / \mathrm{mg}$ de tecido por 24 horas, em temperatura ambiente, para extração do corante.

A concentração de azul de Evans na formamida foi determinada em espectrofotômetro a $620 \mathrm{~nm}$. Os resultados foram comparados com a curva padrão do corante (de 0,5 a 20 $\mu \mathrm{g} / \mathrm{ml}$ ) e expressos como micrograma de azul de Evans / g de tecido seco.

Após a fixação em formol a $10 \%$ por 24 horas, cada fragmento foi incluído em parafina e submetido a corte de 0,5 micra. Os cortes foram montados em lâmina e corados com hematoxilina-eosina.

As lâminas foram enumeradas aleatoriamente de maneira a não permitir sua identificação e examinados por um único patologista.

A escala padronizada de Schmidt et al. ${ }^{17}$ foi utilizada para quantificar o edema histológico, a necrose acinar e o infiltrado inflamatório.

\section{Análise Estatística}

Todos os pâncreas dos ratos dos grupos IC e IIC foram analisados na sua porção proximal e distal ao duodeno, separadamente. A análise estatística foi realizada através do teste t-Student para a comparação dos grupos 2 a 2. Para a análise histológica foi utilizado o teste de Mann-Whitney (para dados não-paramétricos). Para as taxas de mortalidade (grupos IA e IIA, IB e IIB) foi realizado o teste de Kaplan-Meyer. 
$\mathrm{p}<0,05$ foi considerado estatisticamente significante. O programa estatístico Graphpad Prism versão 4.03 (GraphPad Software, San Diego, CA, USA) foi utilizado para a realização dos cálculos.

\section{RESULTADOS}

\section{Subgrupos IA e IIA}

Não houve diferença na taxa de mortalidade entre os grupos.

\section{Subgrupos IB e IIB}

Não houve diferença na taxa de mortalidade entre os grupos.

\section{Subgrupos IC a IIC}

Houve diferença apenas no infiltrado inflamatório dos pâncreas proximais verificados nas lâminas histológicas $(\mathrm{p}=0,043)$. Nenhum dos outros parâmetros analisados mostrou diferença estatística (Figuras 1-6).

\section{DISCUSSÃo}

A pancreatite aguda é doença inflamatória com tratamento predominantemente clínico ${ }^{5}$. Sua fisiopatologia é complexa e envolve uma série de mecanismos diferentes que terminam por levar à ativação precoce das enzimas pancreáticas ${ }^{18-20}$.

Sabe-se que, na pancreatite aguda, ocorre rápida produção de mediadores inflamatórios desencadeando uma resposta inflamatória cuja intensidade se correlaciona com a gravidade das lesões ${ }^{6-8}$.

A temperatura é um importante fator que pode alterar a evolução do processo inflamatório e a resposta inflamatória frente à hipertermia vem sendo objeto de estudos controversos.

Diversos trabalhos apontam uma forte relação entre hipertermia, modulação da expressão de citocinas e resposta do choque térmico ${ }^{21-24}$.

Foi demonstrado que quando a hipertermia é realizada antes da indução da PA leve induzida por injeção de ceruleína , ela protege o ácino pancreático da lesão pela ceruleína ${ }^{25}$. Esta proteção seria mediada pelas HSPs ("heat shock proteins" ou "proteínas do choque térmico") ${ }^{26}$. Acredita-se que as HSPs atuem como chaperonas moleculares facilitando o dobramento final de outras proteínas e protegendoas de danos adicionais. Rakonczay et $a l^{27}$ realizaram um estudo para investigar os efeitos do pré-tratamento com hipotermia e hipertermia na pancreatite aguda grave, induzida por injeção de ácido taurocólico. Os autores notaram um aumento de HSP60 na hipotermia e HSP72 na hipertermia e observaram melhora do edema pancreático e do nível de amilase sérica com a diminuição da temperatura dos ratos, mas não houve melhora nos parâmetros analisados com o aumento da temperatura dos animais. Os autores sugeriram um possível papel protetor da HSP60, mas não da HSP72 na pancreatite aguda grave. Alguns autores sugerem que seu mecanismo protetor está relacionado com sua capacidade de interferir no ativação

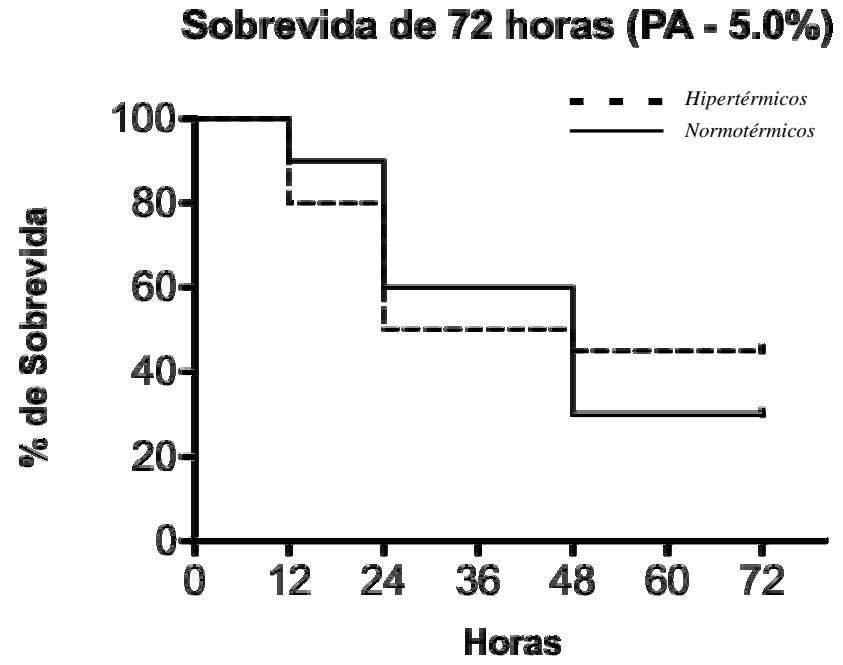

Figura 1 - Efeito da hipertermia na sobrevida de 72 horas, mostrando que não houve diferença nos subgrupos estudados (IA e IIA) ( $p=$ 0.6973).

Sobrevida de 72 horas (PA - 2.5\%)

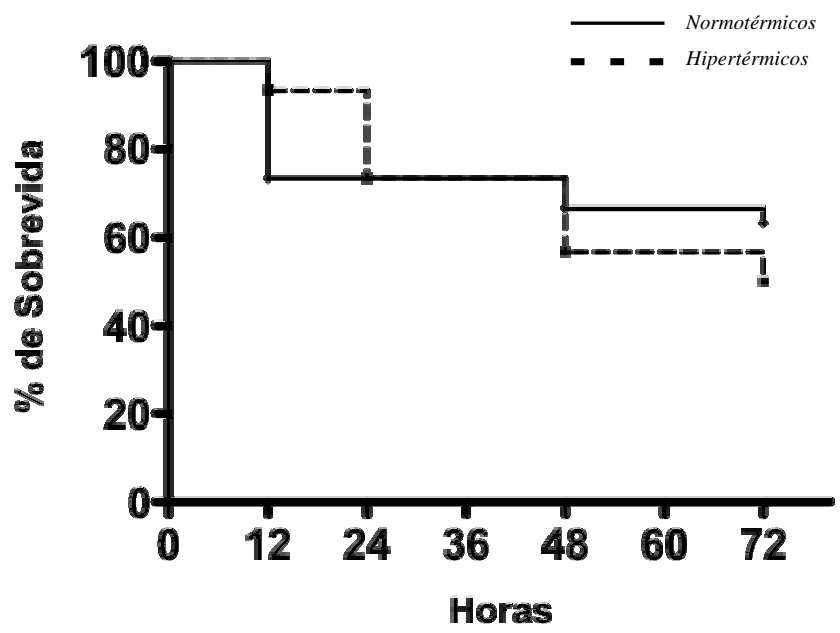

Figura 2 - Efeito da hipertermia na sobrevida de 72 horas, mostrando que não houve diferença nos subgrupos estudados $(I B$ e IIB) $(p=$ 0.4588).

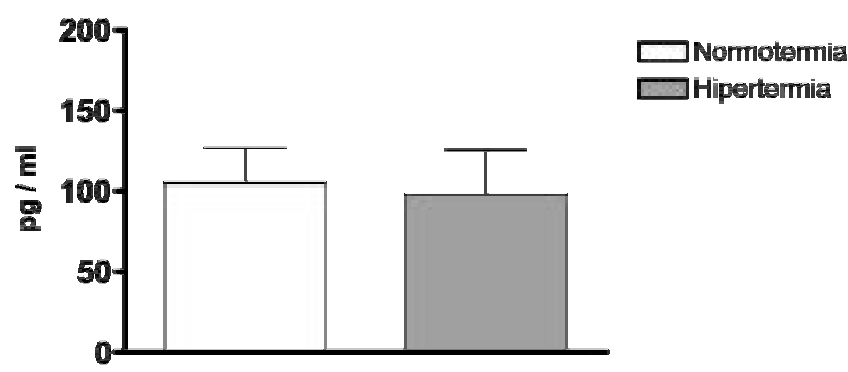

Figura 3 - Efeito da hipertermia nos níveis plasmáticos de IL-6 avaliado pelo método de ELISA, mostrando que não houve diferença nos subgrupos estudados $(p=8264)$.

do NFêB, que é o fator nuclear de transcrição mais importante para a expressão de citocinas inflamatórias como TNF- $\alpha$, IL-1 e IL-6 ${ }^{28,29}$. Após a hipertermia, os níveis séricos dessas citocinas estavam diminuídos. 


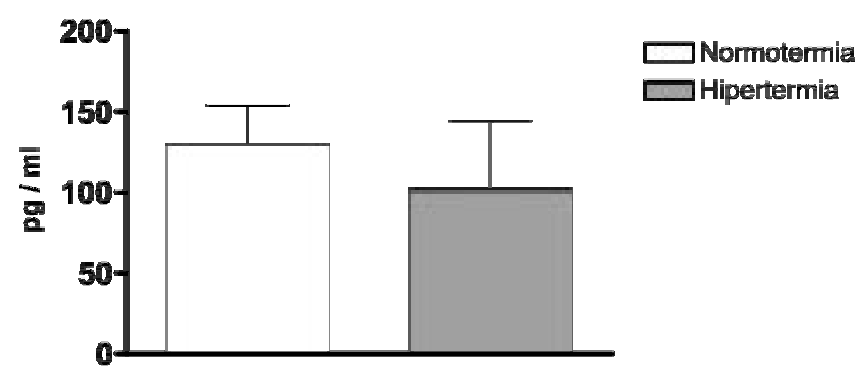

Figura 4 - Efeito da hipertermia nos níveis plasmáticos de IL-10 avaliado pelo método de ELISA, mostrando que não houve diferença nos subgrupos estudados $(p=0.7314)$.

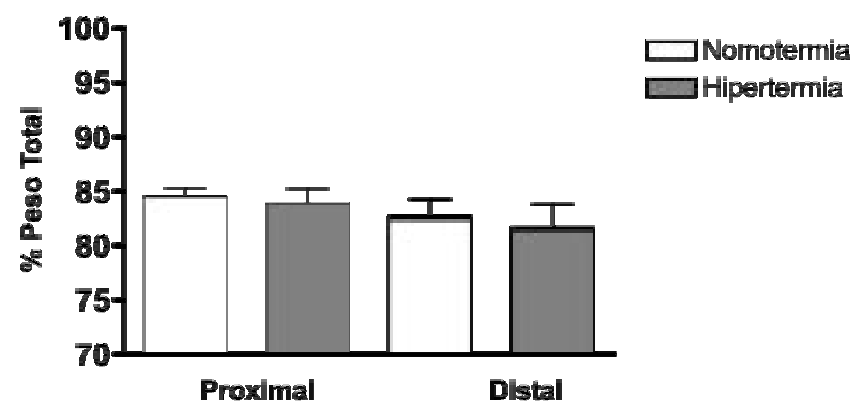

Figura 5 - Efeito da hipertermia no conteúdo de água do tecido pancreático proximal e distal, avaliado pela porcentagem de água livre, demonstrando quantidade semelhante de água nos dois subgrupos avaliados ( $p=0.6946$ e $p=0.7132$, respectivamente).

\section{Permeabllidade Vascular}

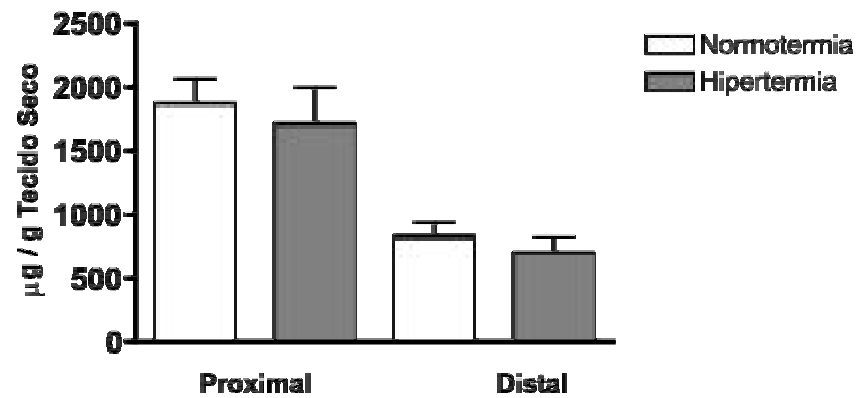

Figura 6 - Efeito da hipertermia na permeabilidade vascular do pâncreas proximal e distal avaliado pelo método do Azul de Evans mostrou permeabilidade vascular semelhante em ambos os subgrupos ( $p=0.6390$ e $p=0.4787$, respectivamente $)$.

Grisé et al..$^{29}$ confirmaram a redução da expressão de algumas citocinas após a hipertermia. Em seu trabalho, camundongos exposto a hipertermia mostram redução dos níveis séricos de IL-6. Este autor postulou que a proteção molecular que as HSPs proporcionam às outras proteínas intracelulares diminui a lesão pancreática e previne a ativação de macrófagos locais assim como a consequente liberação de citocinas inflamatórias. Por sua vez, Ensor et al. ${ }^{30}$ mostraram que a expressão de TNF- $\alpha$ por fagócitos mononucleares foi inibida após o aquecimento dos mesmos in vitro. O mecanismo pelo qual esta ocorre depende da redução da estabilidade do RNAm do TNF- $\alpha$, com consequente desativação precoce da transcrição.

Em recente dissertação de mestrado realizada em nosso meio, Andraus ${ }^{31}$ mostrou piora da pancreatite aguda leve induzida por ceruleína quando ratos com PA são submetidos a hipotermia. Neste trabalho, foi analisada a relação glutationa reduzida / oxidada, a qual se mostrou alterada e permitiu concluir que a hipotermia aumenta os radicais livre de oxigênio no interior da célula acinar e piora o curso clínico da pancreatite. Seguindo um raciocício contrário, realisamos outro recente trabalho ${ }^{32}$ que verificou que a hipertermia reduzia o edema inflamatório na PA leve induzida por ceruleína e; dessa forma, a hipótese de que a hipertermia pudesse alterar também a mortalidade em ratos com PA grave foi cogitada.

O extravazamento do Azul de Evans, a porcentagem de água no tecido pancreático e o estudo histológico de edema com graduação pela escala de Schmidt têm sido utilizados em diversos trabalhos anteriores de nosso grupo $^{5,32,33}$ no sentido de se avaliar o edema inflamatório da PA experimental. É importante ressaltar que a justificativa para se analisar o pâncreas dividido em porção proximal e distal é que a pancreatite aguda induzida por taurocolato não é homogênea e por isso diversas parte dos órgão podem apresentar diferentes níveis de lesão inflamatória.

A necrose acinar e infiltrado inflamatório são outros parâmetros de avaliação histológica do processo inflamatório. No presente estudo apenas o infiltrado inflamatório do pâncreas proximal se encontrou estatisticamente diferente entre os grupos. No entanto não houve diferença estatística nas dosagens de IL-6, IL-10 e de amilase sérica. Os resultados de mortalidade também não mostraram diferença estatística. No prévio estudo de Almeida et al..$^{32}$, foi realizado hipertermia em um grupo de animais sem pancreatite (grupo sham) e nenhuma alteração foi encontrada nos mesmos parâmetros avaliados, justificando assim a não realização de um novo grupo sham no presente trabalho.

Sabe-se que o modelo experimental de pancreatite aguda induzida por taurocolato apresenta taxa de mortalidades diferentes de acordo com a concentração do mesmo (2,5 e $5 \%)^{33}$. Desta forma, dois grupos com diferentes concentrações foram avaliados com o obejtivo de se testar a hipertermia em dois níveis diferentes de gravidade de PA, o que resultou em nenhuma diferença na taxa de mortalidade nos grupos estudados.

O aquecimento não alterou a inflamação pancreática nem a taxa de mortalidade provavelmente porque os mecanismos protetores associados com hipertermia necessitam de um tempo maior para ocorrerem. No entanto, outros estudos são necessários, a fim de elucidar os complexos mecanismos protetores mediados pela hipertermia.

Dessa forma, podemos concluir que a realização de hipertermia provavelmente não traz nenhum benefício não pancreatite aguda grave experimental. 


\begin{abstract}
Objective: The aim of this study is to evaluate the effects of hyperthermia post-treatment on taurocholate-induced severe acute panceatitis (AP) in rats. Method: Severe AP was induced by retrograde injection of $2.5 \%$ or $5 \%$ taurocholate solution into the main pancreatic duct. After the AP induction, animals were heated in a cage with two $100 \mathrm{~W}$ lamps. Body temperature was increased to $39^{\circ} \mathrm{C}$ and maintained at that level for 45 minutes. 72-hours mortality rate, amylase serum levels, histology (edema, acinar necrosis and inflammatory infiltrate), vascular permeability, pancreatic water content and serum levels of IL-6 and IL1 were determinated. Results: Hyperthermia post-treatment on severe AP showed no evidence of alteration in all evaluated parameters. Conclusion: The findings suggest no beneficial effect of the thermal stress on inflammatoy edema and mortality rate in taurocholate AP model.
\end{abstract}

Key words: Pancreatitis; Acute disease; Inflammation; Hyperthermia, induced.

\section{REFERÊNCIAS}

1. Cunha JEM, Machado MCC, Penteado S, Jukemura J, Bacchella T, Pinotti HW. Pancreatic necrosis in Brazil. In: Bradley EL, editor. Acute pancreatitis: diagnosis and therapy. New York: Raven Press; 1994. p. 121-5.

2. Dervenis C, Johnson CD, Bassi C, Bradley E, Imrie CW, McMahon MJ, Modlin I. Diagnosis, objective assessment of severity, and management of acute pancreatitis. Santorini Consensus Conference. Int J Pancreatol. 1999;25(3):195-210.

3. Machado MCC, Bacchella T, Cunha JEM, Jukemura J, Penteado S, Giovanoli ACV, Pinotti HW. Evolução das necroses pancreáticas. Influência do fator infecção. Rev Hosp Clin Fac Med São Paulo. 1985;40(2):120-4.

4. Ranson JHC. Stratification of severity for acute pancreatitis. In: Bradley EL, editor. Acute pancreatitis: diagnosis and therapy. New York: Raven Press; 1994. p. 13-20.

5. Sarner M. Pancreatitis definitons and classification. In: Go VLW, Dimagno EP, Gardner JD, Scheele GA, editors. The pancreas, biology, pathobiology and disease. New York: Raven Press; 1993. p. $575-80$.

6. Formela LJ, Galloway SW, Kingsnorth AN. Inflammatory mediators in acute pancreatitis. Br J Surg. 1995;82(1):6-13.

7. Norman J. The role of cytokines in the pathogenesis of acute pancreatitis. Am J Surg. 1998;175(1):76-83.

8. Norman JG. New approaches to acute pancreatitis: role of inflammatory mediators. Digestion. 1999;60 Suppl 1:S57-60.

9. Rongione AJ, Kusske AM, Kwan K, Ashley SW, Reber HA, McFadden DW. Interleukin 10 reduces the severity of acute pancreatitis in rats. Gastroenterology. 1997;112(3):960-7.

10. Grewal HP, Mohey el Din A, Gaber L, Kotb M, Gaber AO. Amelioration of the physiologic and biochemical changes of acute pancreatitis using an anti-TNF-á polyclonal antibody. Am J Surg. 1994;167(1):214-8; discussion 218-9.

11. Ellingsson HV, Clark PF. The influence of artificial fever on mechanisms of resistance. J Immunol. 1942;43(1):65-83.

12. Jiang Q, De Tolla L, van Rooijen N, Singh IS, Fitzgerald B, Lipsky MM, Kane AS, Cross AS, Hasday JD. Febrile-range temperature modifies early systemic tumor necrosis factor-á expression in mice challenged with bacterial endotoxin. Infection Immun. 1999;67(4):1539-46.

13. Hammami MM, Bouchama A, Al-Sedairy S, Shail E, Alohaly Y, Mohamed GE. Concentrations of soluble tumor necrosis factor and interleukin-6 receptors in heatstroke and heatstress. Crit Care Med. 1997;25(8):1314-9.

14. Lankisch PG, Ihse I. Bile-induced acute experimental pancreatitis. Scand J Gastroenterol. 1987;22(3):257-60.

15. Bernfeld P. Amylase alpha and beta. Methods Enzimol. 1955;1:149-58.
16. Jamieson AD, Pruitt KM, Caldwell RC. An improved amylase assay. J Dent Res. 1969;48(3):483.

17. Schimidt J, Rattner DW, Lewandrowski K, Compton CC, Mandavilli U, Knoefel WT, Warshaw AL. A better model of acute pancreatitis for evaluation therapy. Ann Surg. 1992;215(1):44-56.

18. Montagnini AL, Kubrusly MS, Coelho AMM, Molan NAT, Cunha, JEM, Machado MCC, Pinotti HW. Efeito da administração de omeprazol sobre o conteúdo pancreático de enzimas em ratos. Rev Hosp Clin Fac Med São Paulo. 1995;50(3):272-5.

19. Paran H, Mayo A, Paran D, Neufeld D, Shwartz I, Zissin R, Singer P, Kaplan O, Skornik Y, Freund U. Octreotide treatment in patients with severe acute pancreatitis. Dig Dis Sci. 2000;45(11):2247-51.

20. Salem MZ, Cunha JE, Coelho AM, Sampietri SN, Machado MCC, Penteado S, Abdo EE. Effects of octreotide pretreatment in experimental acute pancreatitis. Pancreatology. 2003;3(2):164-8.

21. Bouchama A, Hammami MM, Al Shail E, De Vol E. Differential effects of in vitro and in vivo hyperthermia on the production of interleukin-10. Intensive Care Med. 2000;26(11):1646-51.

22. Frossard JL, Bhagat L, Lee HS, Hietaranta AJ, Singh VP, Song AM, Steer M, Saluja AK. Both thermal and non-thermal stress protect against caerulein induced pancreatitis and prevent trypsinogen activation in the pancreas. Gut. 2002;50(1):78-83.

23. Norman JG, Fink GW, Messina J, Carter G, Franz MG. Timing of tumor necrosis factor antagonism is critical in determining outcome in murine lethal acute pancreatitis. Surgery. 1996;120(3):515-21.

24. Norman J, Franz M, Messina J, Riker A, Fabri PJ, Rosemurgy AS, Gower WR Jr. Interleukin-1 receptor antagonist decreases severity of experimental acute pancreatitis. Surgery. 1995;117(6):648-55.

25. Wagner AC, Weber H, Jonas L, Nizze H, Strowski M, Fiedler F, Printz H, Steffen H, Göke B. Hyperthermia induces heat shock protein expression and protection against cerulein-induced pancreatitis in rats. Gastroenterology. 1996; 111(5):1333-42.

26. Weber H, Wagner AC, Jonas L, Merkord J, Höfken T, Nizze H, Leitzmann P, Göke B, Schuff-Wener P. Heat shock response is associated with protection against acute interstitial pancreatitis in rats. Dig Dis Sci. 2000;45(11):2252-64.

27. Rakonczay Z Jr, Takacs T, Ivanyi B, Mandi Y, Papai G, Boros I, Varga IS, Jost K, Lonovics J. The effects of hypo and hyperthermic pretreatment on sodium taurocholate-induced acute pancreatitis in rats. Pancreas. 2002;24(1):83-9.

28. Frossard JL, Pastor CM, Hadengue A. Effects of hyperthermia on NF-kappaB binding activity in cerulein-induced acute pancreatitis. Am J Physiol Gastrointest Liver Physiol. 2001;280(6):G1157-62. 
29. Grisé K, Kim F, McFadden D. Hyperthermia induces heat-shock protein expression, reduces pancreatic injury, and improves survival in necrotizing pancreatitis. Pancreas. 2000;21(2):120-5.

30. Ensor JE, Crawford EK, Hasday JD. Warming macrophages to febrile range destabilizes tumor necrosis factor-á mRNA without Inducing heat shock. Am J Physiol. 1995;269(5 Pt 1):C1140-6.

31. Andraus W. Efeito da hipotermia na pancreatite aguda experimental. [Dissertação]. São Paulo (SP): Universidade de São Paulo; 2003.

32. Almeida JL, Jukemura J, Sampietre SN, Patzina RA, Cunha JEM, Machado MCC. Efeito da hipertermia na pancreatite aguda experimental. Arq Gastroenetrol. In press 2007.

33. Mikami Y, Takeda K, Shibuya K, Qiu-Feng H, Egawa S, Sunamura M, Matsuno S. Peritoneal inflammatory cells in acute pancreatitis: relationship of infiltration dynamics and cytokine production with severity of illness. Surgery. 2002;132(1):86-92.
Como citar este artigo:

Yamanari MGI, Kunitake TA, Almeida JLJ, Jukemura J, Cunha JEM, Machado MCC. Efeito da hipertermia na pancreatite aguda grave experimental. Rev Col Bras Cir [periódico na Internet]. 2007;34(1). Disponível em URL: http://ww.scielo.br/rcbc

Endereço para correspondência:

Maurício Gustavo Ieiri Yamanari

Rua Oscar Freire, 1735 - Apto 131

05409-011- São Paulo - SP

Telefone: (55) (11)30836562

E-mail: mauriciogustavo91@yahoo.com.br 